\title{
Intersubband transitions in pseudomorphic InGaAs/GaAs/AIGaAs multiple step quantum wells
}

\author{
H. S. Li, Y. W. Chen, and K. L. Wang \\ Device Research Laboratory, 66-147B Engineering IV, Department of Electrical Engineering, \\ University of California at Los Angeles, Los Angeles, California 90024-1594

\section{Y. C. Lie} \\ Department of Electrical Engineering, M/S 116-81, Caltech, Pasadena, California 91125
}

(Received 29 January 1993; accepted 8 July 1993)

\begin{abstract}
Intersubband transitions from the ground state to the first and second excited states in pseudomorphic $\mathrm{AlGaAs} / \mathrm{InGaAs} / \mathrm{GaAs} / \mathrm{AlGaAs}$ multiple step quantum wells have been observed. The step well structure has a configuration of two AlGaAs barriers confining an InGaAs/GaAs step. Multiple step wells were grown on GaAs substrate with each InGaAs layer compressively strained. During the growth, a uniform growth condition was adopted so that inconvenient long growth interruptions and fast temperature ramps when switching the materials were eliminated. The sample was examined by cross-sectional transmission electron microscopy, an x-ray rocking curve technique, and the results show good crystal quality using this simple growth method. Theoretical calculations were performed to fit the intersubband absorption spectrum. The calculated energies are in good agreement with the observed peak positions for both the $1 \rightarrow 2$ and $1 \rightarrow 3$ transitions.
\end{abstract}

\section{INTRODUCTION}

Intersubband transitions in multiple quantum wells have received a great deal of interest for their applications of novel optoelectronic devices, for example, infrared detectors ${ }^{2,3}$ and modulators. ${ }^{4,5}$ In Ref. 5, infrared modulation was demonstrated utilizing the enhanced Stark shift in multiple $\mathrm{Al}_{x} \mathrm{Ga}_{1-x} \mathrm{As} / \mathrm{GaAs} / \mathrm{Al}_{y} \mathrm{Ga}_{1-y}$ As step quantum well structures. ${ }^{6}$ The device operated at $77 \mathrm{~K}$ because of a large leakage current from thermionic emission. If a higher operation temperature is desired, not only a thicker but also a higher barrier has to be used to block this excessive leakage. However, for the $\mathrm{GaAs} / \mathrm{Al}_{x} \mathrm{Ga}_{1-x}$ As material system, the maximum direct gap barrier height is only 336 $\mathrm{meV}$ with $x=0.45$. For $x$ greater than 0.45 , the $\mathrm{Al}_{x} \mathrm{Ga}_{1-x} \mathrm{As}$ becomes indirect with the $X$ points lower than the $\Gamma$ point. In that case there can be leakage due to the intervalley scattering into the $X$ valleys in the barriers. For the step well, an alternative approach is to use strained InGaAs/GaAs/AlGaAs material combinations since the InGaAs/AlGaAs interface has a larger barrier height.

The strained InGaAs/GaAs/AlGaAs system has been extensively studied in the area of modulation-doped fieldeffect transistors ${ }^{7}$ and lasers. ${ }^{8}$ It is known that, to achieve high electron mobility and sharp photoluminescence peak for this type of structures, different optimum growth conditions have to be employed when growing the InGaAs and $\mathrm{AlGaAs}$ compounds. The $\mathrm{AlGaAs}$ growth requires a higher substrate temperature plus a lower $V$ to III flux ratio, whereas the InGaAs needs a lower temperature and a larger flux ratio. One additional consideration in the growth is the possible formation of misfit dislocations, due to the large lattice mismatch between the InGaAs and GaAs/AIGaAs, once the epitaxial layer exceeds the critical layer thickness. ${ }^{9}{ }^{9} 10$ The fast As flux change when switching the materials can be obtained by installing two As guns or a valved As cell in the growth chamber. To change the substrate temperature, it is a common practice to insert a long growth interruption (about $2 \mathrm{~min}$ ) or a fast temperature ramp in the growth procedure, with only a few exceptions in which a uniform growth temperature is used. For studying doped multiple quantum well structures (with many periods), the repeated temperature changes are inconvenient for the growth. Besides, impurity incorporation during the long interruption may cause doping and contamination problems. It is desired therefore to use a uniform growth condition to grow the entire structure. In this article, we report the growth of pseudomorphic InGaAs/GaAs/AlGaAs multiple step quantum wells on GaAs substrate using this growth condition. The sample was characterized by cross-sectional transmission electron microscopy (TEM) and an $\mathrm{x}$-ray rocking curve technique, and intersubband transitions were demonstrated for the grown sample. The results suggest high quality quantum wells with this simple growth method.

\section{EXPERIMENT}

Samples were grown by a computer controlled PerkinElmer 430 molecular-beam epitaxy (MBE). A $5000 \AA$ GaAs buffer layer, doped with Si to $2.4 \times 10^{18} \mathrm{~cm}^{-3}$, was first grown to obtain a smooth starting surface for the subsequent growth of multiple quantum wells. The multiple quantum well structure contains ten periods of step quantum wells. Each period consists of a $60 \AA \mathrm{In}_{0.22} \mathrm{Ga}_{0.78} \mathrm{As}$ well, a $85 \AA$ GaAs step, and a $267 \AA \mathrm{Al}_{0.37} \mathrm{Ga}_{0.63}$ As barrier. The quantum well was doped with $\mathrm{Si}$ to a total twodimensional (2D) doping level of $5 \times 10^{12} \mathrm{~cm}^{-2}$ for each period. The growth was carried out at a substrate temperature of $530^{\circ} \mathrm{C}$ and with a constant As flux, which gives a $V$ to III flux ratio of about 4 during the InGaAs growth. The multiple quantum wells were capped with a $3000 \AA$ 


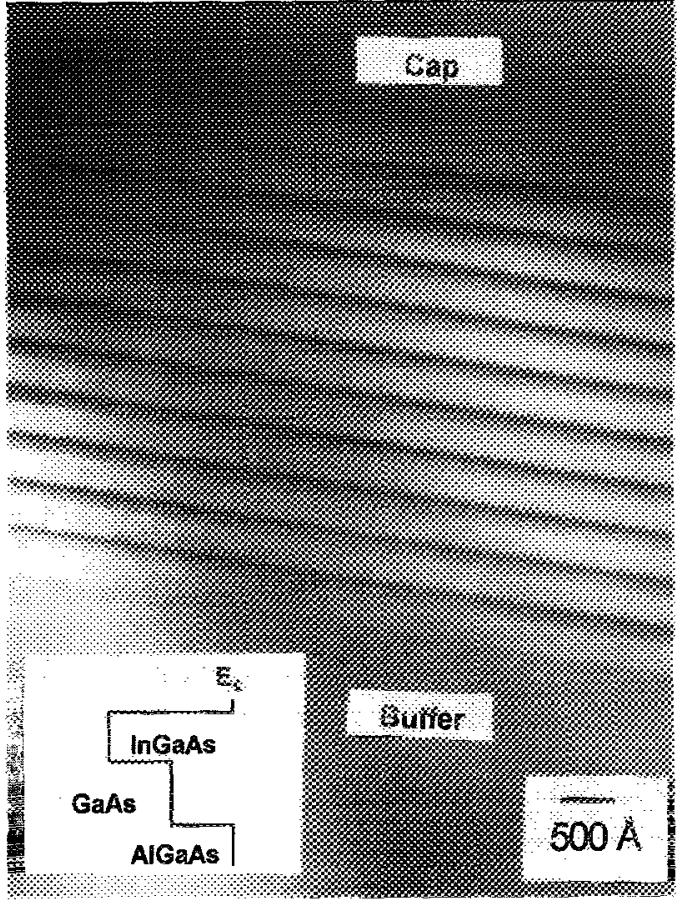

Fig. 1. Cross-sectional TEM micrograph of the sample structure, showing ten periods of the step well, with each InGaAs layer coherently strained on the GaAs buffer. The band diagram is shown inset.

GaAs cap layer, also doped to $2.4 \times 10^{18} \mathrm{~cm}^{-3}$. Intersubband absorption was measured with a Nicolet- 740 Fouriertransform infrared spectrometer (FTIR) at $300 \mathrm{~K}$ using a waveguide scheme. ${ }^{5}$

\section{RESULTS AND DISCUSSION}

Figure 1 shows the cross-sectional TEM micrograph of the multiple step quantum well structure. As one can see, there are a total number of ten period step wells in this structure, with each consisting of three layers, namely the InGaAs, GaAs, and AlGaAs. The band diagram is shown inset. As shown by this picture, no misfit dislocation is observed throughout the quantum well region, indicating that the InGaAs layers are coherently strained to the GaAs buffer. A higher resolution picture was also obtained (not shown) from which the step well was determined to be 66 $\AA$ InGaAs, $94 \AA$ AaAs, $263 \AA$ AlGaAs, and a total period of $423 \AA$. Based on these layer thicknesses, the compositions of the two ternary compounds can be calculated. The small well is $\mathrm{In}_{0.21} \mathrm{Ga}_{0.79} \mathrm{As}$ and the thick barrier is $\mathrm{Al}_{0.29} \mathrm{Ga}_{0.71} \mathrm{As}$. For this sample, the intended indium composition for each InGaAs quantum well is $22 \%$, and hence the multiple quantum well region equivalently contains an average indium content of $3.2 \%$. From a theoretical viewpoint, to prevent the generation of misfit dislocations, the critical layer thicknesses for the individual InGaAs layer as well as the multiple quantum wells have to be considered. ${ }^{11}$ The 60 - $\AA$-thick $\mathrm{In}_{0.22} \mathrm{Ga}_{0.78}$ As quantum well is thinner than the critical layer thickness predicted by either the force or energy balance model, ${ }^{9,10}$ whose calculations give roughly a critical layer thickness of 140 and $460 \AA$, respec-

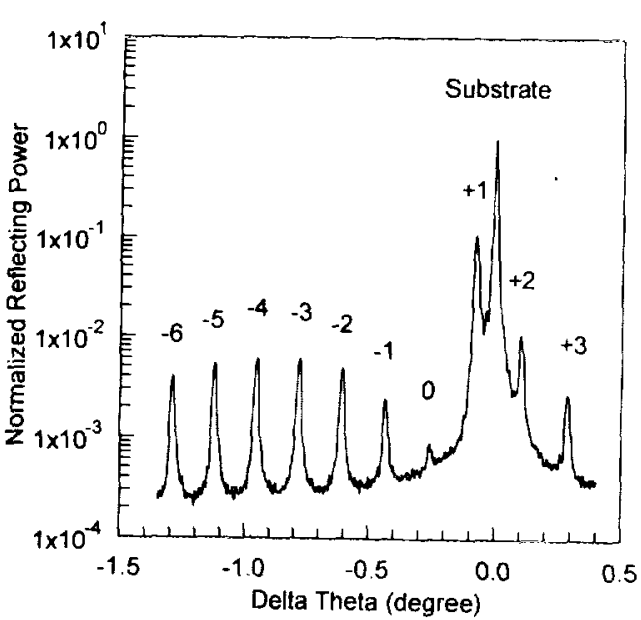

FIG. 2. (400) double crystal $x$-ray rocking curve. The quantum well periodicity and the indium composition were determined to be $423 \AA$ and $22 \%$, respectively.

tively. The total thickness of the multiple quantum wells is about $4120 \AA$. This thickness is slightly above the critical layer thickness of $1400 \AA$ according to the force balance model, nevertheless is still much lower than the value predicted by the energy balance model of $4 \mu \mathrm{m}$. The experimentally determined InGaAs critical layer thickness versus different indium composition was found to be bounded in between these two theoretical predictions. ${ }^{12}$ We believe that the total layer thickness for our structure is below the critical layer thickness limitation, and therefore the structure is fully strained.

The $(400)$ ) double crystal rocking curve of the sample is depicted in Fig. 2. Informations on the quantum well periodicity $(p)$ and perpendicular strain $\left(\left\langle\epsilon^{\perp}\right\rangle\right)$ were obtained through ${ }^{13}$

$$
p=\frac{\lambda\left|\gamma_{H}\right|}{\Delta \theta_{p} \sin 2 \theta_{B}}
$$

and

$$
\left\langle\epsilon^{1}\right\rangle=-\Delta \theta_{0} / \tan \theta_{B},
$$

where $\lambda$ is the $\mathrm{x}$-ray wavelength, $\gamma_{H}$ is the direction cosine of the diffracted waves with respect to the inward normal to the sample surface, $\Delta \theta_{p}$ is the spacing between the multiple quantum well peaks, $\theta_{B}$ is the Bragg angle of the substrate, and $\Delta \theta_{0}$ is the spacing between the quantum well zeroth-order peak and the substrate peak. It is noted that the perpendicular strain in Eq. (2) is defined relative to the substrate and averaged over a quantum well period. Using Eq. (1), the quantum well periodicity was calculated to be $423 \AA$. The average strain for each period was determined to be $0.475 \%$ from Eq. (2). This means an actual strain of $3 \%$ for the InGaAs layer, after divided by the fraction of the InGaAs layer width over the period. To determine the indium composition, the relaxed film lattice mismatch was first deduced for the InGaAs using ${ }^{14}$

$$
\left[\frac{\Delta a}{a}\right]_{r}=\frac{1-v}{1+v}\left[\frac{\Delta a}{a}\right]_{1}+\frac{2 v}{1+v}\left[\frac{\Delta a}{a}\right]_{\|},
$$




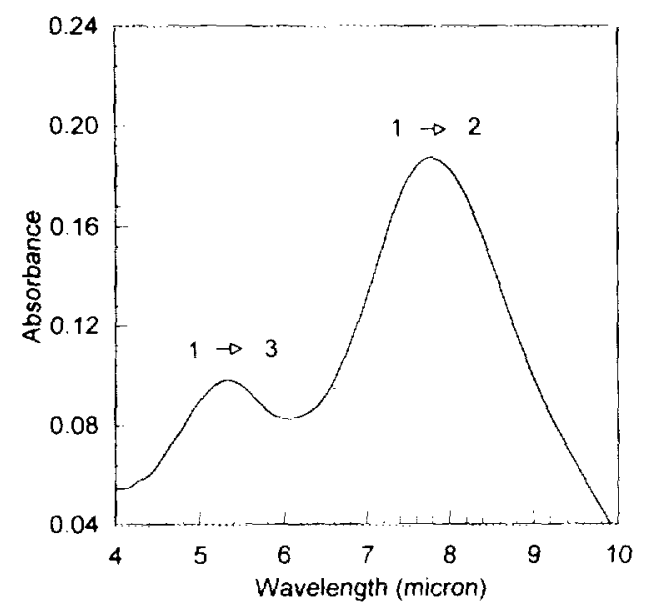

FIG. 3. The absorption spectrum from the FTIR measurement using a waveguide scheme. Both the $1 \rightarrow 2$ and $1 \rightarrow 3$ absorption peaks were observed at 7.6 and $5.3 \mu \mathrm{m}$, respectively.

where $v$ is the Poisson ratio and is 0.311 for the GaAs substrate. By assuming zero parallel strain (i.e., coherently strained), the relaxed film mismatch was calculated to be $1.6 \%$, from which the indium composition was determined via Vegard's law to be $22 \%$. Both the quantum well periodicity and indium composition agree reasonably with the TEM data and calibrations done before the growth.

Depicted in Fig. 3 is the infrared absorption data. Two absorption peaks at $7.6(164 \mathrm{meV})$ and $5.3 \mu \mathrm{m}(232 \mathrm{meV})$ were resolved, and, respectively, assigned to be the $1 \rightarrow 2$ and $1 \rightarrow 3$ transitions in the step well. For convenience, the energy states in the well are sequentially numbered as $1,2,3$, etc. In contrast to the square well case, where the $1 \rightarrow 3$ transition is forbidden by the intersubband sesection rule, this transition was also observed since the symmetry has been broken by the insert of the step in the well. ${ }^{15}$ Both absorption peaks are sensitive to the incident light polarization, and disappear when the polarization is perpendicular to the growth direction. The absorption peak width is $28 \mathrm{meV}$ for the $1 \rightarrow 2$ transition and $36 \mathrm{meV}$ for the $1 \rightarrow 3$ transition. The peaks are broader than those in the case of $\mathrm{GaAs} / \mathrm{AlGaAs}$ step quantum well, in which they are about $20 \mathrm{meV}^{5}$ The reason may be the higher doping level used in this case.

Using the known structural parameters, the osserved intersubband absorption spectrum was fitted with theoretical predictions to determine the InGaAs/GaAs conduction band offset. Structures with various step heights were used to fit the intersubband transition energies. In the calculations, a one-band transfer matrix program ${ }^{16,17}$ was used to calculate the energy states, transition energies, and oscillator strength. The best fitting was obtained for both the $1 \rightarrow 2$ and $1 \rightarrow 3$ transitions by the least square method, with a step height (i.e., the conduction band offset) of 238 $\mathrm{meV}$. The calculated transition energies are $169 \mathrm{meV}$ for the $1 \rightarrow 2$ and $229 \mathrm{meV}$ for the $1 \rightarrow 3$ transition. Both values are close to the experimental data, which are 164 and 232 $\mathrm{meV}$, respectively. Along with the transition energies, the oscillator strengths for the $1 \rightarrow 2$ and $1 \rightarrow 3$ transitions were also calculated to be 0.90 and 0.21 . The $2 \mathrm{D}$ carrier concentration for each period $\rho_{s}$ was then determined from ${ }^{18}$

$$
I_{A}=\rho_{s} N_{T} \frac{e^{2} h}{4 \epsilon_{0} m^{*} c n_{r}} f \frac{\cos ^{2} \theta}{\sin \theta} \cos ^{2} \phi,
$$

where $I_{A}$ is the integration of the absorption strength, $N_{T}$ is the total number of periods that the infrared beam goes through due to the multiple reflections in the waveguide, $f$ is the oscillator strength, $\theta$ is the angle of incidence, and $n_{r}$ is the refractive index. For the structure we used $N_{T}=80$, $I_{A}=65.75 \mathrm{~cm}^{-1}, \theta=45^{\circ}, \phi=0^{\circ}, \rho_{s}$ was calculated to be $5.47 \times 10^{11} \mathrm{~cm}^{-2}$, which is about 0.11 of the dopant level. The discrepancy between the carrier concentration and the dopant level may be due to the strain effect or the AlGaAs related deep levels because the barrier was grown at a low substrate temperature $\left(530^{\circ} \mathrm{C}\right)$. Despite of the fact that the InGaAs/GaAs band offset ratio has been closely investigated by many authors, ${ }^{19}$ as an initial attempt, we estimated the conduction band offset ratio for our sample. Photoluminescence of the sample was performed at $4 \mathrm{~K}$. An excitonic transition from the electron ground state to the lowest heavy hole state was observed at $1.21 \mathrm{eV}$. Assuming an increase of the InGaAs/GaAs conduction band offset by $10 \mathrm{meV}\left(\Delta E_{c}=248 \mathrm{meV}\right.$ ) for the reduced temperature, the conduction band ground state energy was recalculated. The valence band offset was then varied to fit the photoluminescence (PL) peak, and a best fitting was obtained with a valence band offset of $131 \mathrm{meV}$. From these conduction and valence band offset values, the conduction band offset ratio for the InGaAs/GaAs interface was estimated to be $65 \%$.

\section{SUMMARY}

In summary, the growth of pseudomorphic InGaAs/ GaAs/AIGaAs multiple step quantum wells on GaAs substrate has been demonstrated using a single uniform growth procedure. The growth was conducted under a uniform growth condition so that inconvenient long growth interruptions and fast temperature ramps when switching the materials were avoided. Intersubband transitions from both the $1 \rightarrow 2$ and $1 \rightarrow 3$ in the step well were observed, due to the asymmetric shape of the well. The sample structure was also examined by TEM and with $x$-ray rocking curve. The results reveal the step well structure and show good crystal quality. The observed intersubband absorption spectrum was fitted with theoretical calculations. The calculated energies are in good agreement with the observed transition peaks, and the InGaAs/GaAs conduction band offset was deduced. Finally, as an initial attempt, an InGaAs/GaAs conduction band offset ratio was estimated.

\section{ACKNOWLEDGMENTS}

The authors would like to thank Dr. J. M. Tijero for helping the PL mesurements, and Dr. T. W. Kang for providing the TEM data. This work was, in part, supported by DARPA through NCIPT, and by Joint Services Electronics Program. 
${ }^{1}$ L.C. West and S. J. Eglas, Appl. Phys. Lett. 46, 1156 (1985).

${ }^{2}$ B. F. Levine, C. G. Bethea, G. Hasnain, J. Walker, and R. J. Malik, Appl. Phys. Lett. 53, 296 (1988)

${ }^{3}$ C. Hasnain, G. F. Levine, C. G. Bethea, R. As. Logan, J. Walker, and R. J. Malik, Appl. Phys. Lett. 54, 2515 (1989).

${ }^{4}$ A. Harwit and J. S. Harris, Appl. Phys. Lett. 50, 685 (1987)

${ }^{5}$ R. P. G. Karunasiri, Y. J. Mii, and K. L. Wang, IEEE Electron Device Lett. EDL-11, 227 (1990).

${ }^{6}$ Y. J. Mii, R. P. G. Karunasiri, K. L. Wang, and P. F. Yuh, Appl. Phys. Lett. 56, 1986 (1990).

${ }^{7}$ M. V. Baeta Moreira, M. A. Py, M. Gailhanou, and M. Ilegems, J. Vac.

Sci. Technol. B 10, 103 (1992), and references therein.

${ }^{8}$ Stephen D. Offsey, W. J. Schaff, Luke F. Lester, L. F. Eastman, and S.

K. Mckernan, IEEE J. Quantum Electron. QE-27, 1455 (1991), and references therein.

J. W. Mathews and A. E. Blakeslee, J. Cryst. Growth 27, 118 (1974).
${ }^{10}$ R. People and J. C. Bean, Appl. Phys. Lett. 47, 322 (1986).

${ }^{11}$ R. Hull, J. C. Bean, F. Cerdeira, A. T. Fiory, and J. M. Gibson, Appl. Phys. Lett. 48, 56 (1986).

${ }^{12}$ Y. C. Chen, P. K. Bhattacharya, and J. Singh, J. Vac. Technol. B 10, 769 (1992)

${ }^{13}$ V. S. Speriosu and T. Vreeland, Jr., J. Appl. Phys. 56, 1591 (1984)

${ }^{14}$ S. N. G. Chu, A. T. Macrander, K. E. Strege, and W. D. Johnston, Jr., J. Appl. Phys. 57, 249 (1985).

${ }^{15}$ P. F. Yuh and K. L. Wang, J. Appl. Phys. 65, 4377 (1989)

${ }^{16}$ R. Tsu and L. Esaki, Appl. Phys. Lett. 22, 562 (1973).

${ }^{17}$ M. O. Vassell, J. Lee, and H. F. Lockwood, J. Appl. Phys. 54, 5206 (1983).

${ }^{18}$ R. P. G. Karunasiri, J. S. Park, Y. J. Mii, and K. L. Wang, Appl. Phys Lett. 57, 2585 (1990).

${ }^{19}$ B. Jogai, Appl. Phys. Lett. 59, 1329 (1991), and references therein. 